\title{
Iniciação Científica Distribuição Livre Ideal da formiga-leão (Insecta, Neuroptera)
}

\author{
Vitor Gabriel de Oliveira Leite ${ }^{\mathbb{D}}$ \& Tatiane do Nascimento Lima
}

Universidade Federal de Mato Grosso do Sul, Aquidauana, Mato Grosso do Sul, Brasil.

\author{
Entomology Beginners, vol. 2: e014 (2021)
}

\begin{abstract}
Resumo. As larvas de formiga-leão, Myrmeleon brasiliensis (Návas), são insetos que constroem armadilhas em solo seco e arenoso para a captura de suas presas. O objetivo deste trabalho foi observar como os instares larvais de $M$. brasiliensis distribuem-se dentro de um modelo de Distribuição Livre Ideal. Neste trabalho foi observado que $M$. brasiliensis, ao serem expostas a uma situação que simula um micro-habitat com manchas que recebem alimento e manchas que não recebem alimento, as larvas de todos os instares ocuparam todo o espaço, independente da maior ou menor oferta de alimento. A ocorrência de canibalismo e construção de uma armadilha afastada das larvas vizinhas foram os fatores que guiaram a distribuição das larvas M. brasiliensis.
\end{abstract}

Palavras-chave: Forrageamento ótimo; Myrmeleontidae; predador senta-espera.

\section{Ideal Free Distribution of antlion (Insecta, Neuroptera)}

Abstract. Antlion larvae Myrmeleon brasiliensis (Návas) are insect that build traps on dry, sandy soil to capture their prey. The objective of this work was to observe how the larval instars of $M$. brasiliensis are distributed within an Ideal Free Distribution model. In this work it was observed that $M$. brasiliensis, when exposed a micro-habitat with patchs that receive food and patchs that do not receive food, the larvae of all instars occupied the entire space, regardless of the greater or lesser supply of food. The occurrence of cannibalism and the construction of a trap away from neighboring larvae were the factors that guided the distribution of $M$. brasiliensis larvae.

Keywords: Myrmeleontidae; optimal foraging; predator sits-waiting.

As larvas de Neuroptera (Myrmeleontidae) conhecidas como formiga-leão constroem armadilhas em forma de funil no solo seco e arenoso para a captura de suas presas. A singularidade dessa estratégia de forrageamento há muito tempo atrai a atenção para esses insetos (SCHARF et al., 2010). A larva permanece quieta no fundo da armadilha, com as mandíbulas abertas, esperando a presa cair. Dado que a armadilha é basicamente um cone invertido escavado em solo de baixa granulometria, seu projeto tem uma função dupla. Primeiro a armadilha canaliza a presa para o fundo do poço, onde se encontra a formiga-leão. E segundo, retarda a fuga da presa porque a areia é instável, grãos escorregam para o fundo da armadilha enquanto a presa tenta fugir (LUCAS, 1982; FRANKS et al., 2019).

Essas larvas comumente são observadas agrupadas em áreas protegidas da ação direta da chuva, do sol e do pisoteio de outros animais. O agrupamento das larvas ocorre por consequência da oviposição do adulto (GOTELLI, 1993). Entretanto, durante seu desenvolvimento as larvas tendem a afastar-se umas das outras para minimizar os efeitos da competição por recursos e da interferência de uma larva sobre a outra (FARJI-BRENER et al., 2020; OVADIA et al., 2020).

As formigas-leão passam por três instares antes da formação da pupa, e emergência dos adultos. $O$ tamanho das armadilhas

\section{Editado por:}

Rodrigo Souza Santos

\section{Histórico Editoral:}

Recebido em: 13.05.2021

Aceito em: 20.07.2021

Publicado em: 04.08 .2021 aumenta com o desenvolvimento larval, o que garante maior sucesso na captura de presas (MISSIRIAN et al., 2006). As larvas menores apresentam um menor sucesso na captura de presas, quando comparado com as larvas maiores de terceiro instar (NONATO e LIMA, 2011). Dessa maneira, no início do desenvolvimento as larvas passam uma fase mais crítica, pois a taxa de captura de presas é baixa. Isso faz com que essas larvas possuam menos energia para investir em tamanho de armadilha e no deslocamento em busca de locais com alta oferta de recurso (ALCALAYA et al., 2014).

De acordo com o modelo de Distribuição Livre Ideal (DLI) os animais de diversas espécies se comportam na competição pela exploração de regiões em busca de recursos, distribuindose por estes espaços a fim de alcançar o máximo de recursos disponível (FRETWELL e LUCAS, 1970; TREGENZA, 1995; LOEHLE, 2012). Dentro dos agrupamentos, as larvas de formiga-leão maiores tendem a apresentar um maior sucesso na captura de presas, sendo assim as larvas menores deveriam deslocar-se em busca de áreas longe da interferência das larvas maiores. Entretanto, como as formigas, principal presa, evitam forragear nas áreas ocupadas por formigas-leão, as larvas maiores (por possuírem maior reserva energética e por suportarem a ação direta do sol) é que tendem a deslocar-se para fora dos agrupamentos (GOTELLI, 1996; MILER et al., 2018; LIMA et al., 2019). Sendo assim, as manchas com maior oferta de recurso

\section{Autor Correspondente:}

Tatiane do Nascimento Lima tatianenlima@gmail.com

Agência(s) de Fomentos:

Nenhum Agência de fomento declarada 
estariam ocupadas pelas larvas maiores.

Dentro de um modelo de DLI para as larvas da espécie Myrmeleon brasiliensis Návas (Neuroptera: Myrmeleontidae), além da busca pelo recurso, o tamanho das larvas também deve guiar a distribuição espacial desses organismos. O objetivo deste trabalho foi observar como os instares larvais de M. brasiliensis distribuem-se dentro de um modelo de Distribuição Livre Ideal. Foram avaliados: I) a abundância de larvas em áreas com e sem oferta de alimento; II) a probabilidade de as larvas maiores ocuparem a área com oferta de alimento; III) a ocorrência de canibalismo e IV) o tamanho das armadilhas no começo e no fim do experimento.

As larvas $M$. brasiliensis foram coletadas em uma Área de Proteção Permanente (160 ha) situada no município de

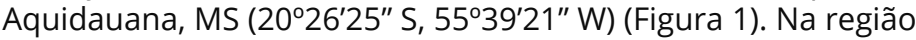
a vegetação predominante é do tipo savana arbórea aberta (Cerrado), com algumas inclusões do tipo savana arbórea densa (cerradão). O clima da região, segundo a classificação descrita por Köppen-Geiger é do tipo Aw (Tropical de Savana), com precipitação média anual de $1200 \mathrm{~mm}$ e temperaturas médias de $26,2^{\circ} \mathrm{C}$, com inverno seco e verão chuvoso.

Para avaliar a distribuição dos instares larvais de $M$. brasiliensis em resposta à oferta de recurso, as larvas foram coletadas e levadas ao laboratório de Estudos da Biodiversidade da Universidade Federal de Mato Grosso do Sul. Foram montados dois experimentos, no experimento I (controle) seis larvas do mesmo tamanho (segundo instar) foram colocadas no centro de um recipiente de plástico $(40 \mathrm{~cm} \times 20 \mathrm{~cm} \times 8 \mathrm{~cm})$ contendo areia do próprio local da coleta. No experimento II duas larvas de diferentes tamanhos (duas de cada instar) (total de 6 larvas) foram colocadas no centro de um recipiente de plástico (40 $\mathrm{cm} \times 20 \mathrm{~cm} \times 8 \mathrm{~cm}$ ). Nos dois experimentos, após 24 horas, tempo dado para todas as larvas construírem suas armadilhas, foi registrado o local onde cada larva construiu sua armadilha (Figura 1).

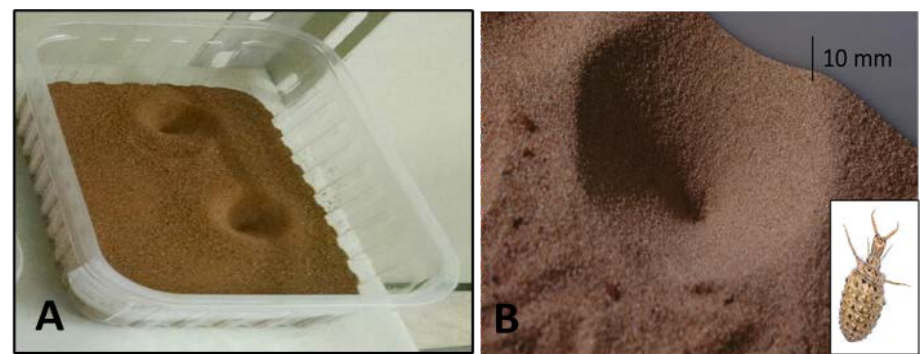

Figura 1. (A) Experimento em andamento, podendo ser observado duas armadilhas de $M$. brasiliensis e (B) detalhe de uma armadilha e de uma larva de M. brasiliensis (Fotos: Vitor Gabriel de Oliveira Leite).

A partir daí, foram ofertadas como presas formigas cortadeiras (Hymenoptera) em apenas um dos lados do recipiente. A oferta ocorreu de maneira manual, uma formiga foi ofertada dentro de cada armadilha que estava do lado direito do recipiente. Os experimentos seguiram por cinco dias. Diariamente fol registrado o local que cada larva estava construindo sua armadilha, o tamanho das armadilhas, a distância entre as armadilhas e a ocorrência de canibalismo. Todas as medidas foram realizadas com paquímetro digital (precisão 0,01 mm).

Cada experimento foi replicado 10 vezes, totalizando 120 larvas de $M$. brasiliensis. A abundância de larvas nas áreas de menor e maior recurso foi comparada através de teste T. No experimento II, a probabilidade das larvas maiores (instar 3) estarem na área de maior oferta de recurso foi analisada por Regressão Logística. O tamanho das armadilhas no começo e no final dos Experimentos I e II foi comparado através de Teste T e Wilcoxon, respectivamente. Todas as análises foram realizadas no software livre MyStat (https://systatsoftware. com/downloads/download-mystat/).

No Experimento I (controle), com larvas M. brasiliensis do mesmo tamanho (segundo instar) após os cinco dias de observação, a média ( \pm desvio padrão) de larvas observadas no lado do recipiente com oferta de alimento foi de $2,3( \pm 0,82)$. No lado do recipiente onde não ocorreu a oferta de alimento a média de larvas foi de $2,7( \pm 0,67)$. Não foi observada diferença significativa no número de larvas em cada lado do recipiente ( $\mathrm{t}$ $=-0,93 ; p=0,37$ ).

Nesse experimento foi observado canibalismo entre as larvas. A média de ocorrência de canibalismo entre as amostras foi de $17 \%$. O canibalismo ocorreu somente no primeiro dia do experimento, quando as larvas se deslocavam para a construção de suas armadilhas. Ou seja, as larvas que primeiro construíram suas armadilhas predaram aquelas que ainda estava deslocando-se em busca de um local para forragear.

Foi observado um aumento significativo no tamanho das armadilhas durante os cinco dias do experimento ( $t=-5,97$; $G L$ $=47 ; p=0,01)$. O tamanho médio das armadilhas construídas pelas larvas de $M$. brasiliensis foi de $32,13( \pm 8,86) \mathrm{mm}$ no primeiro dia e de $41,21( \pm 9,54) \mathrm{mm}$ no último dia. A distância média entre as armadilhas foi de $117 \mathrm{~mm}$. As larvas mais próximas e mais distantes construíram suas armadilhas separadas por $56 \mathrm{~mm}$ e $252 \mathrm{~mm}$, respectivamente.

No Experimento II, com larvas de $M$. brasiliensis do primeiro, segundo e terceiro instar, o número de larvas nos dois lados do recipiente também não diferiu $(t=1,49 ; p=0,63)$. No lado sem alimento o número médio de larvas foi de $2,7( \pm 1,33)$, enquanto no lado com alimento o número médio de larvas foi de 2,3 $( \pm 1,33)$. Dessa maneira as larvas foram observadas ocupando todo o espaço e não apenas a área que recebeu alimento.

A taxa de canibalismo entre as larvas de $M$. brasiliensis foi de 13\%. Da mesma maneira que o observado no Experimento I, o canibalismo ocorreu logo no primeiro dia do experimento, quando as larvas estavam deslocando-se para construir suas armadilhas. Não houve diferença entre o tamanho das larvas na ocupação de um dos lados do recipiente $\left(X^{2}=1.78 ; G L=1 ; p=\right.$ $0.18)$. Ou seja, a probabilidade das larvas de todos três instares ocuparem o lado do recipiente com oferta de alimento foi a mesma.

Foi observado um aumento significativo no tamanho das armadilhas $(z=2,37 ; p=0,01)$ durante o experimento, sendo de 38,86 $( \pm 11,77) \mathrm{mm}$ no início e de $47,50( \pm 13,23) \mathrm{mm}$ no fim do experimento. A distância entre as armadilhas construídas foi de $128 \mathrm{~mm}$. As larvas mais próximas e mais distantes construíram suas armadilhas separadas por $62 \mathrm{~mm}$ e $268 \mathrm{~mm}$, respectivamente.

Assim, neste trabalho foi observado que as larvas de $M$. brasiliensis ao serem expostas a uma situação que simula um micro-habitat com manchas que recebem alimento e manchas que não recebem alimento, ocuparam todo o espaço, independente da maior ou menor oferta de alimento. Este padrão foi observado tanto no experimento com larvas do mesmo tamanho, quanto no experimento com larvas de diferentes tamanhos (instar um, dois e três).

Embora não tenha sido observada uma maior probabilidade de as larvas maiores ocuparem as manchas com maior oferta de recurso, a decisão de construir a armadilha logo no início do experimento e a ocorrência do canibalismo guiaram a distribuição das larvas. Dessa maneira, dentro de um modelo de Distribuição Livre Ideal para as larvas de $M$. brasiliensis a decisão de construir a armadilha e o canibalismo devem guiar a distribuição espacial desses organismos. A presença de canibais pode induzir organismos menores de uma mesma espécie a evitar forragear em áreas próximas a grandes indivíduos (BIRO et al., 2003; RUDOLF, 2006). Dessa maneira, o canibalismo estruturado pelo tamanho do predador, acaba exercendo uma forte influência na dinâmica da população (CLAESSEN et al., 2000).

Um dos pressupostos da teoria da Distribuição Livre Ideal é que 
indivíduos escolhem o habitat que lhes confere o maior fitness. No caso das larvas de $M$. brasiliensis observadas neste trabalho, aquelas larvas que primeiro construíram suas armadilhas apresentaram vantagens sobre as outras. Como no primeiro momento toda a área disponível era homogênea em relação à oferta de recurso, a primeira variável que afetou a distribuição das larvas foi a presença das larvas vizinhas. A seleção de habitat é uma decisão individual e cada organismo deve fazer uma escolha baseada não só na qualidade do habitat, mas também nos custos e benefícios do uso de uma determinada área (FRETWELL e LUCAS, 1970). Avaliar as diferenças individuas na seleção de um habitat pode ajudar a entender a razão dos organismos escolhem habitats de menor qualidade (LOEHLE, 2012).

Após a construção das armadilhas foi observado que o estabelecimento das larvas foi mantido até o final do experimento. De maneira geral, as formigas-leão exibem forte fidelidade ao local de construção de suas armadilhas, visto que a realocação da armadilha é energeticamente custosa (CROWLEY e LINTON, 1999; LIMA e LOPES, 2016). Ademais, os predadores de emboscada investem energia na construção de uma armadilha para captura de presas e poupam energia ao evitar custo envolvido na sua procura (LUCAS, 1985). No ambiente natural o alimento afeta o deslocamento das larvas que passam por longos períodos sem capturar suas presas (SCHARF e OVADIA, 2006). Entretanto, em intervalos curtos o movimento das larvas ocorre devido a uma resposta denso dependente causada pela competição direta e não pela falta absoluta de alimento.

Tratando-se do tamanho da armadilha, foi observado um aumento no tamanho delas durante o experimento, o que pode estar relacionado ao consumo de presas (tanto ofertadas, quanto provenientes do canibalismo). O diâmetro e a profundidade das armadilhas das formigas-leão são características que influenciam o sucesso da captura, afetando a probabilidade de encontro e de retenção das presas (LOMASCOLO e FARJIBRENER, 2001). Arnett e Gotelli (2001) observaram que larvas de formiga-leão com limitação de alimento constroem e reparam seus poços com mais frequência do que as larvas bem alimentadas.

Neste trabalho foi observado que larvas de $M$. brasiliensis ao serem expostas à uma situação que simula um micro-habitat com manchas que recebem alimento e manchas que não recebem alimento, todas as larvas ocuparam todo o espaço, independente da maior ou menor oferta de alimento. Dentro de um modelo de Distribuição Livre Ideal a competição intraespecífica (ocorrência de canibalismo e construção de uma armadilha afastada das larvas vizinhas) foi o fator que guiou a distribuição das larvas de M. brasiliensis.

\section{Referências}

ARNETT, A.; GOTELLI, N. Pit-building decisions of larval ant lions: effects of larval age, temperature, food and population source. Journal of Insect Behavior, v. 14, n. 1, p. 89-97, 2001. DOI: https://doi.org/10.1023/A:1007853730317

ALCALAYA, Y.; BARKAEA, E. D.; OVADIA, O.; SCHARF, I. Consequences of the instar stage for behavior in a pit-building antlion. Behavioural Processes, v. 103, p. 105-111, 2014. DOI: https://doi.org/10.1016/j.beproc.2013.11.009

BIRO, P. A.; POST, JR.; PARKINSON, E. A. From individuals to populations: prey fish risk-taking mediates mortality in wholesystem experiments. Ecology, v. 84, n. 9, p. 2419-2431, 2003. DOI: https://doi.org/10.1890/02-0416
CROWLEY, P. H.; LINTON, M. P. Antlion foraging: tracking prey across space and time. Ecology, v. 80, n. 7, p. 2271-2282, 1999. DOI: https://doi.org/10.1890/0012-9658(1999)080[2271:AFTPA S]2.0.CO;2

CLAESSEN, D.; ROOS, A. M.; PERSSON, L. Dwarfs and giants: cannibalism and competition in sizestructured populations. American Naturalist, v. 155, n. 2, p. 219-237, 2000. DOI: https://.org/10.1086/303315

FRETWELL, S. D.; LUCAS, H. L. On territorial behavior and other factors influencing habitat distribution in birds. Acta Biotheoretica, v. 19, p. 16-36, 1970. DOI: https://doi.org/10.1007/BF01601953

FRANKS, N. R.; WORLEY, A.; FALKENBER, M.; SENDOVAFRANKS, A. B.; CHRISTENSEN, K. Digging the optimum pit: antlions, spirals and spontaneous stratification. Proceedings of the Royal Society B, v. 286, p. 20190365, 2019. DOI: https://doi.org/10.1098/rspb.2019.0365

FARJI-BRENER, A. G.; JUNCOSA-POLZELLA, A. S.; TEJADA, D. M.; CENTENO-ALVARADO, D.; HERNÁNDEZ-SOTO, M.; SOTOHUAIRA, M.; GUTIÉRREZ-CRUZ, S. Disadvantages of living in a populous neighborhood for sit-andwait predators: competition for space reduces pit-trap size in antlion larvae. Ethology, v. 126, n. 6. p. 1-7, 2020. DOI: https://doi.org/10.1111/eth.13079

GOTELLI, N. J. Ant Lion zones: causes of high-density predator aggregations. Ecology, v. 74, n. 1, p. 226-237, 1993. DOI: https://doi.org/10.2307/1939517

GOTELLI, N. J. Ant community structure: effects of predatory ant lions. Ecology, v. 77, n. 2, p. 630-638, 1996. DOI: https://doi.org/10.2307/2265636

LUCAS, J. R. The biophysics of pit construction by antlion larvae (Myrmeleon, Neuroptera). Animal Behavior, v. 30, n. 3, p. 651664, 1982. DOI: https://doi.org/10.1016/S0003-3472(82)80135-8

LUCAS, J. R. Metabolic rates and pit-construction costs of two antlion species. Journal of Animal Ecology, v. 54, n. 1, p. 295309, 1985. DOI: https://doi.org/10.2307/4639

LOEHLE, C. Conditional choice model of habitat selection explains the source-sink paradox. Ecological Modelling, v. 235/236, p. 59-66, 2012. DOI: https://doi.org/10.1016/j. ecolmodel.2012.03.037

LIMA, T. N.; LOPES, F. S. Effect of density, disturbance and food on displacement of the Myrmeleon brasiliensis (Navás, 1914) (Neuroptera, Myrmeleontidae). Ecología Austral, v. 26, n. 2, p. 166-170, 2016. DOI: https://doi.org/10.25260/EA.16.26.2.0.168

LIMA, T. N.; FREIRE, L. G.; LIMA, D. C. A. Effect of asymmetric competition on distance among Myrmeleon brasiliensis (Návas, 1914) (Neuroptera: Myrmeleontidae) larvae. Acta Scientiarum Biological Sciences, v. 41, e45871, 2019. DOI: https://doi.org/10.4025/actascibiolsci.v41i1.45871

LOMÁSCOLO, S.; FARJI-BRENER, A. G. Adaptive short-term changes in pit design by antlion larvae (Myrmeleon sp.) in 
response to different prey conditions. Ethology Ecology \& Evolution, v. 13, n. 4, p. 393-397, 2001. DOI: https://doi.org/10. 1080/08927014.2001.9522770

MISSIRIAN, G. B.; UCHÔA-FERNANDES, M. A.; FISCHER, E. Development of Myrmeleon brasiliensis (Navás) (Neuroptera, Myrmeleontidae), in laboratory, with different natural diets. Revista Brasileira de Zoologia, v. 23, n. 4, p. 1044-1050, 2006. DOI: https://doi.org/10.1590/S0101-81752006000400009

MILER, K.; YAHAYA, B.; CZARNOLESKI, M. Different predation efficiencies of trap-building larvae of sympatric antlions and wormlions from the rainforest of Borneo. Ecological Entomology, v. 43, n. 2, p. 255-262, 2018. DOI: https://doi.org/10.1111/een.12495

NONATO, L. M.; LIMA, T. N. El comportamiento de predación de los estadios larvales de Myrmeleon brasiliensis (Neuroptera, Myrmeleontidae). Revista Colombiana de Entomología, v. 37, n. 1, p. 354-356, 2011.

OVADIA, O.; SCHARF, I.; BARKAE, E. D.; LEVI, T.; ALCALAY,
Y. Asymmetrical intra-guild predation and niche differentiation in two pit-building antlions. Israel Journal of Ecology and Evolution, v. 66, n. 1/2, p. 82-90, 2020. DOI: https://doi.org/10.1163/22244662-20191067

RUDOLF, V. H. The influence of size-specific indirect interactions in predator-prey systems. Ecology, v. 87, n. 2, p. 362-371, 2006. DOI: https://doi.org/10.1890/05-0961

SCHARF, I.; OVADIA, O. Factors influencing site abandonment and site selection in a sit-and-wait predator: a review of pitbuilding antlion larvae. Journal Insect Behaviour, v. 19, n. 2, p. 197-218, 2006. DOI: https://doi.org/10.1007/s10905-006-9017-4

SCHARF, I.; BARKAE, E. D.; OVADIA, O. Response of pitbuilding antlions to repeated unsuccessful encounters with prey. Animal Behaviour, v. 79, n. 1, p. 153-158, 2010. DOI: https://doi.org/10.1016/j.anbehav.2009.10.017

TREGENZA, T. Building on the ideal free distribution. Advances in Ecological Research, v. 26, p. 253-302, 1995. DOI: https://doi.org/10.1016/S0065-2504(08)60067-7 